\title{
ON THE NATURAL REPRESENTATION OF THE SYMMETRIC GROUPS
}

\author{
by H. K. FARAHAT \\ (Received 29 March, 1961)
}

1. Introduction. Let $E$ be an arbitrary (non-empty) set and $S$ the restricted symmetric group on $E$, that is the group of all permutations of $E$ which keep all but a finite number of elements of $E$ fixed. If $\Phi$ is any commutative ring with unit element, let $\Gamma=\Phi(S)$ be the group algebra of $S$ over $\Phi, \Gamma \supset \Phi$; and let $M$ be the free $\Phi$-module having $E$ as $\Phi$-base. The " natural" representation of $S$ is obtained by turning $M$ into a $\Gamma$-module in the obvious manner, namely by writing for $\alpha \in S, \lambda_{i} \in \Phi$,

$$
\alpha \sum_{i \in E} \lambda_{i} i=\sum_{i \in E} \lambda_{i} \alpha i
$$

Our object is a more or less complete analysis of this representation. It turns out that the situation is particularly simple when $E$ is an infinite set, the natural representation being indecomposable independently of the ring of scalars $\Phi$; furthermore when $\Phi$ is a field the natural representation contains only one non-trivial irreducible constituent $(\S 6)$. The case in which the number $n$ of elements of $E$ is finite occupies $\$ \$ 2-5$, where the natural representation is seen as one of a set of representations obtained from extensions by $\Phi$ of a certain submodule $M_{0}$ of $M$. Most of these representations are indecomposable ( $(4)$; and when $\Phi$ is a field they have only one non-trivial irreducible constituent of degree $n-1$ or $n-2$ depending on the characteristic of the field. In this way one obtains a non-trivial modular irreducible representation for each symmetric group $(\S 5)$.

2. The natural representation of the symmetric group. Let $\Phi$ be a commutative ring with unit element, $S$ a group, and $\Gamma=\Phi(S)$ the group algebra of $S$ over $\Phi$; we shall use 1 to denote the neutral element of $S$ as well as the unit element of $\Phi$. By a representation module of $S$ over $\Phi$ we shall understand a (left) $\Gamma$-module $M$ which is $\Phi$-free, i.e. which has a $\Phi$-base. This last requirement is automatically satisfied when $\Phi$ is a field, since every module over a field is necessarily free. By choosing a $\Phi$-base for $M$ we can construct a matrix representation of $S$ in the usual manner.

Since the ring $\Phi$ is commutative, any two $\Phi$-bases of a $\Phi$-free module have the same number of elements $\dagger$ and consequently any two matrix representations arising from the same representation module have the same "degree". The usual notions of homomorphism, isomorphism, etc., of representation modules also obtain. For instance the representation module $M$ is said to be decomposable if it is possible to express $M$ as the direct sum of two proper representation submodules. Note that $M$ may be decomposable as a $\Gamma$-module, but not as a representation module. Similarly $M$ is a simple or irreducible representation module if and only if the only representation submodule of $M$ apart from itself is the null submodule.

$\dagger$ See N. Bourbaki, Algèbre, Ch. II §1, p. 20, Ex. 13, and Ch. III §5, No. 7, Cor. 2, p. 67. 
Throughout $\S 2-5$ we take $S$ to be the symmetric group on a finite set $E$; for $M$ we take the natural representation module of $S$ described in the introduction. Let $n$ be the number of elements of $E$.

Clearly the ring $\Phi$ is itself a $\Phi$-free module (having $\Phi$-base consisting of the unit element 1 by itself). We make $\Phi$ into a representation module for $\Gamma$ by writing, for $\alpha \in S, \phi \in \Phi, \alpha \phi=\phi$. The matrix representation corresponding to this module is the unit-representation $\alpha \rightarrow 1$. We now define a mapping $v: M \rightarrow \Phi$ by the equation

$$
v\left(\sum_{i \in E} \lambda_{i} i\right)=\sum_{i \in E} \lambda_{i}
$$

Obviously, $v$ is a $\Phi$-homomorphism, and we may verify that if $\alpha \in S$ then $\alpha v=v \alpha$, and $v$ is in fact a $\Gamma$-homomorphism. Put $M_{0}=\operatorname{Ker} v$; thus $M_{0}$ consists of all elements $\sum \lambda_{i} i$ of $M$ for which $\sum \lambda_{i}=0$.

Clearly $M_{0}$ is a $\Gamma$-submodule of $M$. However, it is also a representation submodule of $M$. To show this, it is enough to exhibit a $\Phi$-base for $M_{0}$; and it is an easy matter to verify that if $i_{1}$ is a chosen element of $E$ then the $n-1$ elements $i-i_{1}\left(i \neq i_{1}, i \in E\right)$ constitute such a base.

By the first isomorphism theorem it follows that $M$ is an extension of $M_{0}$ by the "trivial " module $\Phi$. The question arises as to whether or not this extension splits, i.e. whether $M_{0}$ is a direct summand of $M$. We prove

(2.1) TheOREM. $M_{0}$ is a direct summand of $M$ if and only if $n \Phi=\Phi$.

Proof. Suppose that $n \Phi=\Phi$, that is that $n n^{\prime}=1$ for a suitable $n^{\prime} \in \Phi$. Let $e$ denote the sum of all elements of $E: e=\sum_{i \in E} i$, and define the mapping $v^{\prime}: \Phi \rightarrow M$ by

$$
v^{\prime} \phi=n^{\prime} \phi e .
$$

Since $\alpha e=e$ for all $\alpha \in S, \nu^{\prime}$ is a $\Gamma$-homomorphism. We have, for $\phi \in \Phi$,

$$
\nu v^{\prime} \phi=v n^{\prime} \phi e=n^{\prime} \phi n=\phi ;
$$

whence $v v^{\prime}$ is the identity map of $\Phi$. It follows that $\xi=v^{\prime} v$ is an idempotent $\Gamma$-endomorphism of $M$ into itself:

We have

$$
\check{\zeta}^{2}=\left(v^{\prime} v\right)^{2}=v^{\prime} v v^{\prime} v=v^{\prime} v
$$

$$
\xi \sum \lambda_{i} i=v^{\prime}\left(\sum \lambda_{i}\right)=n^{\prime}\left(\sum \lambda_{i}\right) e
$$

and this is zero if and only if $\sum \lambda_{i}=0$, i.e. if and only if $\sum \lambda_{i} i \in M_{0}$. It follows that $M_{0}$ is a direct summand of $M$, more precisely, that $M$ is the direct sum of $M_{0}$ and the "trivial" cyclic submodule $\Phi e$ generated by $e$.

Conversely if $M_{0}$ is a direct summand of $M$ then there exists a $\Gamma$-epimorphism $\pi: M \rightarrow M_{0}$ whose restriction to $M_{0}$ is the identity map. Let $i_{1}$ be a fixed element of $E$ and put

$$
\pi i_{1}=\sum_{j \in E} \lambda_{j} j
$$


so that we have $\sum \lambda_{j}=0$. Since $\pi$ is a $\Gamma$-homomorphism we have for any $\alpha \in S, \alpha \pi=\pi \alpha$; hence

$$
\sum_{j \in E} \lambda_{j} \alpha j=\alpha \pi i_{1}=\pi \alpha i_{1}=\pi\left(\left(\alpha i_{1}-i_{1}\right)+i_{1}\right)=\left(\alpha i_{1}-i_{1}\right)+\pi i_{1} .
$$

If $j, j^{\prime}$ are distinct elements of $E$ other than $i_{1}$, there exists $\alpha \in S$ such that $\alpha j^{\prime}=j, \alpha i_{1}=i_{1}$. Comparing coefficients of $j$ in $(*)$ we then get $\lambda_{j^{\prime}}=\lambda_{j}$. It follows that the coefficient of $j$ $\left(j \neq i_{1}\right)$ in $\pi i_{1}$ is independent of $j$. Let $\lambda$ be this coefficient. Then $\lambda_{i_{1}}=-(n-1) \lambda$ and so

$$
\pi i_{1}=\lambda\left(e-n i_{1}\right), \quad e=\sum_{i \in E} i
$$

Equation (*) now gives for arbitrary $\alpha$,

i.e.

$$
\begin{gathered}
\lambda\left(e-n \alpha i_{1}\right)=\alpha \pi i_{1}=\alpha i_{1}-i_{1}+\lambda\left(e-n i_{1}\right), \\
-\dot{\lambda} n \alpha i_{1}=\alpha i_{1}-i_{1}(1+\lambda n) .
\end{gathered}
$$

Choosing for $\alpha$ any permutation which "moves" $i_{1}$ and comparing coefficients of $\alpha i_{1}$, we get $-\lambda n=1$. Hence $n \Phi=\Phi$ and the proof is complete.

3. The group of extensions of $M_{0}$ by $\Phi$. The module $A$ is said to be an extension of the module $B$ by the module $C$ if $B$ is a submodule of $A$ such that $A / B$ is isomorphic with $C$. Here we are concerned with the extensions of $M_{0}$ by $\Phi$.

According to the general theory of extensions of representation modules (see e.g. D. G. Northcott, Homological algebra, Ch. $10, \S 9$ ) all extensions of $M_{0}$ by $\Phi$ will be known once the cocycles on $S$ into $\mathrm{Hom}_{\Phi}\left(\Phi, M_{0}\right) \approx M_{0}$ are determined. We recall that a cocycle on $S$ into $M_{0}$ is a mapping $\varepsilon: S \rightarrow M_{0}$ such that

$$
\varepsilon(\alpha \beta)=\alpha \varepsilon(\beta)+\varepsilon(\alpha) \quad(\alpha, \beta \in S) .
$$

Those extensions which are isomorphic with the direct sum of $M_{0}$ and $\Phi$ (i.e. split extensions) correspond to the coboundaries, namely cocycles of the form

$$
\varepsilon_{0}(\alpha)=\alpha m_{0}-m_{0} \quad(\alpha \in S)
$$

where $m_{0}$ is a fixed element of $M_{0}$. The group of extensions $\operatorname{Ext}\left(M_{0}, \Phi\right)$ is then defined as the factor group of the additive group of cocycles modulo the subgroup of coboundaries.

In this section we compute the group of extensions of $M_{0}$ by $\Phi$. Let $i_{1}, i_{2}, \ldots, i_{n}$ be the elements of the set $E$ in any order, and, if $r$ is any integer and $1 \leqq k \leqq n-1$, write $\tau_{k+r(n-1)}$ $=\left(i_{k}, i_{n}\right)$. Then it is well known (see e.g. R. C. Carmichael, Groups of finite order, Ch. VII $\S 48$ ) that $\tau_{1}, \ldots, \tau_{n-1}$ generate the symmetric group $S$ on $E$, and that every identical relation between them is a consequence of the following relations:

$$
\text { (a) } \tau_{k}^{2}=1,(b)\left(\tau_{k} \tau_{k+1}\right)^{3}=1, \quad(c)\left(\tau_{k} \tau_{k+1} \tau_{k} \tau_{j}\right)^{2}=1 \text {, }
$$

where $k, j$ range over the set $1,2, \ldots, n-1$, except that $j$ is different from $k$ and $k+1$.

It follows that a cocycle $\varepsilon$ is uniquely determined once its "values" at $\tau_{1}, \ldots, \tau_{n-1}$ are known. In fact, if $\omega_{1}, \omega_{2}, \ldots, \omega_{q}$ is any selection of the above 2-cycles, then

$$
\varepsilon\left(\omega_{1} \omega_{2} \ldots \omega_{q}\right)=\omega_{1} \omega_{2} \ldots \omega_{q-1} \varepsilon\left(\omega_{q}\right)+\omega_{1} \omega_{2}, \ldots \omega_{q-2} \varepsilon\left(\omega_{q-1}\right)+\ldots+\omega_{1} \varepsilon\left(\omega_{2}\right)+\varepsilon\left(\omega_{1}\right) .
$$


Using this formula we can verify easily from $(a),(b),(c)$ that the values of the cocycle at $\tau_{1}, \ldots, \tau_{n-1}$ satisfy the identities

$\left(a^{\prime}\right)\left(1+\tau_{k}\right) \varepsilon\left(\tau_{k}\right)=0$,

(b') $\left(1+\tau_{k} \tau_{k+1}+\tau_{k+1} \tau_{k}\right)\left\{\varepsilon\left(\tau_{k}\right)+\tau_{k} \varepsilon\left(\tau_{k+1}\right)\right\}=0$,

(c') $\left(\tau_{j}+\tau_{k} \tau_{k+1} \tau_{k}\right)\left[\varepsilon\left(\tau_{j}\right)+\tau_{j}\left(\tau_{k} \tau_{k+1}+1\right) \varepsilon\left(\tau_{k}\right)+\tau_{k} \tau_{k+1} \varepsilon\left(\tau_{k+1}\right)\right]=0$,

where, once more, $k, j$ range over $1,2, \ldots, n-1, j$ being different from $k$ and $k+1$.

Conversely, suppose that we are given $n-1$ elements $\varepsilon\left(\tau_{1}\right), \ldots, \varepsilon\left(\tau_{n-1}\right)$ of $M_{0}$ which satisfy the relations $\left(a^{\prime}\right),\left(b^{\prime}\right),\left(c^{\prime}\right)$. We show that they are the values of a cocycle at $\tau_{1}, \ldots, \tau_{n-1}$. If $\omega_{1}, \ldots, \omega_{q}$ is a sequence of elements $\tau_{k}$, put

$$
\varepsilon\left(\omega_{1}, \omega_{2}, \ldots, \omega_{q}\right)=\sum_{r=1}^{q} \omega_{1} \omega_{2} \ldots \omega_{r-1} \varepsilon\left(\omega_{r}\right),
$$

it being understood that empty sums are zero. It follows immediately that, if $\omega_{1}^{\prime}, \ldots, \omega_{p}^{\prime}$ is another sequence, then

$$
\varepsilon\left(\omega_{1}, \ldots, \omega_{q}, \omega_{1}^{\prime}, \ldots, \omega_{p}^{\prime}\right)=\omega_{1} \omega_{2} \ldots \omega_{q} \varepsilon\left(\omega_{1}^{\prime}, \ldots, \omega_{p}^{\prime}\right)+\varepsilon\left(\omega_{1}, \ldots, \omega_{q}\right) .
$$

Since the $\varepsilon\left(\tau_{k}\right)$ satisfy $\left(a^{\prime}\right),\left(b^{\prime}\right),\left(c^{\prime}\right)$, it follows that $\varepsilon\left(\omega_{1}, \omega_{2}, \ldots, \omega_{q}\right)=0$ whenever $\omega_{1} \omega_{2} \ldots \omega_{q}$ $=1$. Hence, if $\omega_{1} \omega_{2} \ldots \omega_{q}=\omega_{1}^{\prime} \omega_{2}^{\prime} \ldots \omega_{p}^{\prime}$, then $\varepsilon\left(\omega_{1}, \ldots, \omega_{q}, \omega_{p}^{\prime}, \ldots, \omega_{1}^{\prime}\right)=0$ and so

$$
\begin{aligned}
\varepsilon\left(\omega_{1}, \omega_{2}, \ldots, \omega_{q}\right) & =-\left(\omega_{1} \omega_{2} \ldots \omega_{q}\right) \varepsilon\left(\omega_{p}^{\prime}, \omega_{p-1}^{\prime}, \ldots, \omega_{1}^{\prime}\right) \\
& =-\left(\omega_{1}^{\prime} \omega_{2}^{\prime} \ldots \omega_{p}^{\prime}\right) \varepsilon\left(\omega_{p}^{\prime}, \omega_{p-1}^{\prime}, \ldots, \omega_{1}^{\prime}\right) \\
& =\varepsilon\left(\omega_{1}^{\prime}, \omega_{2}^{\prime}, \ldots, \omega_{p}^{\prime}\right),
\end{aligned}
$$

by $\left(a^{\prime}\right)$ and the definition. Thus we may define for $\alpha=\omega_{1} \omega_{2} \ldots \omega_{q}, \varepsilon(\alpha)=\varepsilon\left(\omega_{1}, \omega_{2}, \ldots, \omega_{q}\right)$, and then $\varepsilon$ is a cocycle on $S$ into $M_{0}$ as required. Hence we have proved

(3.1) Lemma. If $\varepsilon$ is a cocycle on $S$ into $M_{0}$, then the elements $m_{k}=\varepsilon\left(\tau_{k}\right)(1 \leqq k \leqq n-1)$ of $M_{0}$ satisfy the relations $\left(a^{\prime}\right),\left(b^{\prime}\right),\left(c^{\prime}\right)$. Conversely, any $n-1$ elements of $M_{0}$ satisfying these relations are the values of a cocycle at $\tau_{1}, \ldots, \tau_{n-1}$.

Let $\varepsilon$ be a cocycle, and for $k=1,2, \ldots, n-1$ put

$$
\varepsilon\left(\tau_{k}\right)=\sum_{l=1}^{n} \phi_{k, i} i_{l}
$$

Applying $\left(a^{\prime}\right)$ we find that

$$
\phi_{k, k}=-\phi_{k, n}=\phi_{k} \quad \text { (say) }
$$

and

$$
2 \phi_{k, l}=0 \text { for } l \neq k, l \neq n \text {. }
$$

Next apply $\left(b^{\prime}\right)$, comparing coefficients of $i_{n}$ on both sides. We have

$$
0=\text { sum of coefficients of } i_{n}, i_{k}, i_{k+1} \text { in } \varepsilon\left(\tau_{k}\right)+\tau_{k} \varepsilon\left(\tau_{k+1}\right)=\phi_{k, k+1}+\phi_{k+1, k} \text {. }
$$

Hence

$$
\phi_{k, k+1}=\phi_{k+1, k} \text {. }
$$


Finally apply $\left(c^{\prime}\right)$, comparing coefficients of $i_{j}$ on both sides. Then $0=$ sum of coefficients of $i_{n}, i_{j}$ in

$$
\varepsilon\left(\tau_{j}\right)+\tau_{j}\left(\tau_{k} \tau_{k+1}+1\right) \varepsilon\left(\tau_{k}\right)+\tau_{k} \tau_{k+1} \varepsilon\left(\tau_{k+1}\right)
$$

The contribution of the first term is zero by (i). The contribution of the second is the sum of the coefficients in $\varepsilon\left(\tau_{k}\right)$ of $\tau_{k+1} \tau_{k} \tau_{j} i_{n}, \tau_{k+1} \tau_{k} \tau_{j} i_{j}, \tau_{j} i_{n}, \tau_{j} i_{j}$, which is clearly equal to

$$
\phi_{k, j}+\phi_{k, k}+\phi_{k, j}+\phi_{k, n}=2 \phi_{k, j}+\phi_{k}-\phi_{k}=0 \text {. }
$$

The contribution from the last term is obviously the sum of the coefficients in $\varepsilon\left(\tau_{k+1}\right)$ of $\tau_{k+1} \tau_{k} i_{n}, \tau_{k+1} \tau_{k} i_{j}$; that is

$$
\phi_{k+1, k}+\phi_{k+1, j}=0 \text {. }
$$

Hence, by (iii) and (iv), we see that, for each $k, \phi_{k, l}$ has constant value provided that $l \neq k$, $l \neq n$. Denote this common value by $\psi_{k}$; then we have proved that

$$
\varepsilon\left(\tau_{k}\right)=\psi_{k} \sum_{l \neq k, n} i_{l}+\phi_{k}\left(i_{k}-i_{n}\right) \quad\left(2 \psi_{k}=0\right),
$$

or writing as usual $e=i_{1}+i_{2}+\ldots+i_{n}$, we have

$$
\varepsilon\left(\tau_{k}\right)=\psi_{k} e+\xi_{k}\left(i_{k}-i_{n}\right) \text {. }
$$

Since $\varepsilon\left(\tau_{k}\right) \in M_{0}$, we have $n \psi_{k}=0=2 \psi_{k}$. Let $\Phi_{0}$ denote the set of elements $\psi$ of $\Phi$ such that $(2, n) \psi=0$. Thus $\Phi_{0}$ is necessarily zero if $n$ is odd. Then $\psi_{k} \in \Phi_{0}$ (all $k$ ).

Now a straightforward calculation shows that for arbitrary $\xi_{k} \in \Phi$, the elements

$$
m_{k}=\xi_{k}\left(i_{k}-i_{n}\right) \quad(k=1,2, \ldots, n-1)
$$

satisfy the requirements of Lemma (3.1). Hence there is a unique cocycle $\varepsilon^{\prime}$ such that $\varepsilon^{\prime}\left(\tau_{k}\right)$ $=\xi_{k}\left(i_{k}-i_{n}\right)$. It follows from (3.2) that the equations

$$
\varepsilon^{\prime \prime}\left(\tau_{k}\right)=\psi_{k} e \quad(k=1,2, \ldots, n-1)
$$

also define a cocycle. Clearly for any $\alpha \in S, \varepsilon^{\prime \prime}(\alpha)=\psi_{\alpha} e$, where $\psi_{\alpha} \in \Phi_{0}$. We have for $\alpha, \beta \in S$,

Hence

$$
\psi_{\alpha \beta} e=\varepsilon^{\prime \prime}(\alpha \beta)=\alpha \varepsilon^{\prime \prime}(\beta)+\varepsilon^{\prime \prime}(\alpha)=\alpha \cdot \psi_{\beta} e+\psi_{\alpha} e=\left(\psi_{\beta}+\psi_{\alpha}\right) e
$$

$$
\psi_{\alpha \beta}=\psi_{\alpha}+\psi_{\beta},
$$

and so either $\psi_{\alpha}=0$ (all $\alpha$ ), or else there is a non-zero element $\psi \in \Phi_{0}$ such that

$$
\psi_{\alpha}= \begin{cases}0 & \text { if } \alpha \text { is even } \\ \psi & \text { if } \alpha \text { is odd }\end{cases}
$$

This last case is only possible when $n$ is even.

Conversely it is clear that, if $n$ is even and $\psi \in \Phi_{0}$, then

is a cocycle on $S$ into $M_{0}$.

$$
\varepsilon^{\prime \prime}(\alpha)= \begin{cases}0 & \text { if } \alpha \text { is even, } \\ \psi e & \text { if } \alpha \text { is odd }\end{cases}
$$


Thus we have completely determined the cocycles:

Every cocycle $\varepsilon$ is given by

$$
\varepsilon\left(\tau_{k}\right)=\psi e+\xi_{k}\left(i_{k}-i_{n}\right) \quad(1 \leqq k \leqq n-1),
$$

where $\psi \in \Phi_{0}, \xi_{k} \in \Phi$; and conversely if, $\psi \in \Phi_{0}, \xi_{k} \in \Phi$, then (3.3) defines a cocycle on $S$ into $M_{0}$.

Remark. We observe that for $n>2$ the elements $\psi, \xi_{1}, \ldots, \xi_{n-1}$ are uniquely determined by $\varepsilon\left(\tau_{1}\right), \ldots, \varepsilon\left(\tau_{n-1}\right)$. For $n=2$ we have $\psi e=\psi\left(i_{1}+i_{2}\right)=\psi\left(i_{1}-i_{2}\right)$, so that $\psi$ may be replaced by zero. This will be assumed to be the case whenever an interpretation of (3.3) for $n=2$ is required in what follows.

Now we determine the coboundaries. If

where

$$
\begin{gathered}
\varepsilon(\alpha)=\alpha m_{0}-m_{0}, \\
m_{0}=\sum_{k=1}^{n} \mu_{k} i_{k} \quad\left(\sum_{k=1}^{n} \mu_{k}=0\right)
\end{gathered}
$$

is any coboundary, then

$$
\varepsilon\left(\tau_{k}\right)=\left(\mu_{n}-\mu_{k}\right)\left(i_{k}-i_{n}\right)=\xi_{k}\left(i_{k}-i_{n}\right),
$$

say, where $\xi_{k}=\mu_{n}-\mu_{k}$; we have

$$
\xi_{1}+\ldots+\xi_{n-1}=(n-1) \mu_{n}-\mu_{1}-\ldots-\mu_{n-1}=n \mu_{n} \in n \Phi .
$$

Conversely, suppose that $\varepsilon$ is a cocycle such that

$$
\varepsilon\left(\tau_{k}\right)=\xi_{k}\left(i_{k}-i_{n}\right) \quad\left(\xi_{1}+\ldots+\xi_{n-1} \in n \Phi\right) .
$$

Put $\xi_{1}+\ldots+\xi_{n-1}=n \mu_{n}$ and define $\mu_{1}, \ldots, \mu_{n-1}$ by

$$
\mu_{k}=\mu_{n}-\xi_{k} \quad(1 \leqq k \leqq n-1) .
$$

If $m_{0}=\sum_{k=1}^{n} \mu_{k} i_{k}$, then

$$
v\left(m_{0}\right)=\mu_{1}+\ldots+\mu_{n}=n \mu_{n}-\left(\xi_{1}+\ldots+\xi_{n-1}\right)=0,
$$

so that $m_{0} \in M_{0}$; and furthermore

Consequently

$$
\tau_{k} m_{0}-m_{0}=\left(\mu_{n}-\mu_{k}\right)\left(i_{k}-i_{n}\right)=\xi_{k}\left(i_{k}-i_{n}\right)=\varepsilon\left(\tau_{k}\right) .
$$

$$
\varepsilon(\alpha)=\alpha m_{0}-m_{0} \quad(\text { for } \alpha \in S)
$$

and $\varepsilon$ is a coboundary. Hence we have

(3.4) The cocycle (3.3) is a coboundary if and only if

$$
\psi=0 \text { and } \xi_{1}+\ldots+\xi_{n-1} \in n \Phi .
$$

We are now in a position to prove

(3.5) THEOREM. If $n>2$, the group $\operatorname{Ext}\left(M_{0}, \Phi\right)$ of extensions of $M_{0}$ by $\Phi$ is isomorphic to the direct sum of $\Phi / n \Phi$ and $\Phi_{0}$, where $\Phi_{0}$ is the set of elements $\psi$ of $\Phi$ such that $(2, n) \psi=0$. For $n=2$ we have $\operatorname{Ext}\left(M_{0}, \Phi\right) \approx \Phi / 2 \Phi$. 
If $n>2$ and $\varepsilon$ is the cocycle (3.3), put $N \varepsilon=\left(\overline{\xi_{1}+\ldots+\xi_{n-1}}, \psi\right)$. Then $N$ maps the (additive) group of cocycles homomorphically onto $\Phi / n \Phi \oplus \Phi_{0}$. Here $\bar{\xi}$ denotes the residue class of $\xi$ modulo $n \Phi$. The kernel of $N$ is precisely the group of coboundaries, by the remark preceding the theorem. This proves the first assertion and makes the second assertion obvious.

(3.6) CoRollary. If $n \Phi=\Phi$ then every extension of $M_{0}$ by $\Phi$ splits.

For in this case we have $\Phi / n \Phi=0, \Phi_{0}=0$, and so $\operatorname{Ext}\left(M_{0}, \Phi\right)=0$.

4. The extensions of $M_{0}$ by $\Phi$. As before, $M$ denotes the $\Phi$-free module with base $E=\left\{i_{1}, i_{2}, \ldots, i_{n}\right\}$. We shall show how $M$ can be made into a $\Gamma$-module in such a way that it becomes an extension of $M_{0}$ by $\Phi$ having a prescribed cocycle $\varepsilon$. If $\alpha$ is any element of $\Gamma$, we shall use $\alpha \varepsilon$ to denote the $\Phi$-homomorphism of $M$ into itself corresponding to $\alpha$ in the proposed module. Furthermore, we denote the resulting $\Gamma$-module by $\varepsilon M$. Clearly it is sufficient to define $\alpha \varepsilon$ when $\alpha \in S$. We write, for $m \in M, \alpha \in S$,

$$
\alpha \varepsilon m=\alpha m+\nu(m)(\varepsilon-\bar{\varepsilon})(\alpha),
$$

where $\bar{\varepsilon}$ is the cocycle of the natural module $M$ (computed by using (4.3) below) and, as before, $v(m)$ is the sum of coefficients of $m$. Clearly, when $\varepsilon=\bar{\varepsilon},(4.1)$ reduces to the natural definition $\alpha \varepsilon m=\alpha m$. We verify that (4.1) does in fact make $M$ into a $\Gamma$-module. If $\alpha, \beta \in S$, then, for $m \in M$,

$$
\begin{aligned}
& (\alpha \beta) \varepsilon m-\alpha \varepsilon(\beta \varepsilon m)=(\alpha \beta) m+v(m)(\varepsilon-\bar{\varepsilon})(\alpha \beta)-\alpha \varepsilon[\beta m+v(m)(\varepsilon-\bar{\varepsilon})(\beta)] \\
& \quad=(\alpha \beta) m+v(m)(\varepsilon-\bar{\varepsilon})(\alpha \beta)-\alpha[\beta m+v(m)(\varepsilon-\bar{\varepsilon})(\beta)]-v[\beta m+v(m)(\varepsilon-\bar{\varepsilon})(\beta)](\varepsilon-\bar{\varepsilon})(\alpha) \\
& \quad=v(m)\{(\varepsilon-\bar{\varepsilon})(\alpha \beta)-\alpha(\varepsilon-\bar{\varepsilon})(\beta)-(\varepsilon-\bar{\varepsilon})(\alpha)\}=0,
\end{aligned}
$$

since $\varepsilon-\bar{\varepsilon}$ is a cocycle. This proves our contention.

(4.2) THEOREM. The representation module $\varepsilon M$ defined by (4.1) is an extension of $M_{0}$ by $\Phi$ with cocycle $\varepsilon$.

Proof. By (4.1), we have $\alpha \varepsilon m=\alpha m$ whenever $m \in M_{0}$. Hence $M_{0}$ is a $\Gamma$-submodule of $\varepsilon M$. Furthermore, we have for $m \in M, \alpha \in S$,

$$
v(\alpha \varepsilon m)=v(\alpha m)=\alpha v(m) ;
$$

hence $v$ is a $\Gamma$-epimorphism of $\varepsilon M$ onto $\Phi$. Since $M_{0}=\operatorname{Ker} v$, it follows that $\varepsilon M$ is an extension of $M_{0}$ by $\Phi$. It remains only to compute the cocycle of this extension; and to do this we need mappings which express $M$ as a direct sum of the $\Phi$-modules $M_{0}$ and $\Phi$. Define $\eta: M \rightarrow M_{0}$, $\rho: \Phi \rightarrow M$ by

$$
\eta m=m-v(m) i_{n}, \quad \rho \phi=\phi i_{n},
$$

where $m \in M, \phi \in \Phi$. It is easy to see that

$$
M_{0} \stackrel{\text { incl }}{\rightarrow} M \stackrel{\eta}{\rightarrow} M_{0} ; \quad \Phi \stackrel{\rho}{\rightarrow} M \stackrel{\nu}{\rightarrow} \Phi
$$


is a representation of $M$ as a direct sum of the $\Phi$-modules $M_{0}$ and $\Phi$. According to the usual method for computing the cocycle $\varepsilon_{1}$ of the extension $\varepsilon M$ we have, for $\alpha \in S$,

$$
\begin{aligned}
& \varepsilon_{1}(\alpha)=\eta \alpha \varepsilon \rho 1=\eta \alpha \varepsilon i_{n}=\eta\left[\alpha i_{n}+(\varepsilon-\bar{\varepsilon})(\alpha)\right], \\
& \varepsilon_{1}(\alpha)=\alpha i_{n}-i_{n}+(\varepsilon-\bar{\varepsilon})(\alpha) .
\end{aligned}
$$

But $\bar{\varepsilon}$ is the cocycle of the "natural" module $M$. Hence when $\varepsilon=\bar{\varepsilon}$ we have $\varepsilon_{1}=\bar{\varepsilon}$. Consequently our formula gives

$$
\bar{\varepsilon}(\alpha)=\alpha i_{n}-i_{n}
$$

and $\varepsilon_{1}(\alpha)=\varepsilon(\alpha)$; that is, the cocycle of the extension $\varepsilon M$ computed by using (4.3) is $\varepsilon$. The proof is complete.

Remark. The proof also yielded the formula (4.4) for the cocycle of the natural extension of $M_{0}$ by $\Phi$ computed by using the representation (4.3). In particular we have $\bar{\varepsilon}\left(\tau_{k}\right)=i_{k}-i_{n}$. By (3.4), we have that $\bar{\varepsilon}$ is a coboundary if and only if $n-1 \in n \Phi$, i.e. if and only if $n \Phi=\Phi$. This yields another proof of Theorem (2.1).

The remainder of this section will be devoted to a discussion of the decomposability of these extensions $\varepsilon M$ as representation modules. We begin with an easy lemma.

(4.5) Lemma. If the representation module $A$ is decomposable, then there exists an idempotent endomorphism $\delta$ of $A$ such that $\delta$ and 1 are linearly independent over $\Phi$, that is, such that

$$
\phi_{1} \delta a+\phi_{2} a=0 \text { for all } a \in A \text { implies that } \phi_{1}=\phi_{2}=0 \text {. }
$$

Proof. Suppose that $B$ and $C$ are proper representation submodules of $A$ such that $A=B+C$ (direct sum). Every element $a \in A$ has a unique expression in the form $a=b+c$ with $b \in B, c \in C$. Let $\delta$ be the mapping of $A$ into itself carrying every element $a$ to its component $b$ in $B$. Obviously $\delta$ is an idempotent endomorphism of $A$, and it only remains to show that $\delta, 1$ are linearly independent over $\Phi$. Now since $B$ is a representation module, it is $\Phi$-free, and so the only element $\phi \in \Phi$ such that $\phi B=0$ is the zero element. A similar assertion holds for $C$. However $B=\delta A$, and $C=(1-\delta) A$. It follows that $\phi \delta=0$ implies that $\phi=0$ and that $\phi(1-\delta)=0$ also implies that $\phi=0$. Suppose now that $\phi_{1} \delta+\phi_{2} 1=0$. Multiplying by $1-\delta$ and remembering that $\delta^{2}=\delta$, we get $\phi_{2}(1-\delta)=0$ and hence $\phi_{2}=0, \phi_{1}=0$. The lemma follows.

(4.6) Lemma. If $n>2$ and $\delta \in \operatorname{Hom}_{\Gamma}\left(\varepsilon M, \varepsilon^{\prime} M\right)$, then $\delta M_{0} \subset M_{0}$. This conclusion is valid in the case $n=2$ provided that no non-zero element of $\Phi_{0}$ annihilates $\xi_{1}^{\prime}$.

Proof. We have, for $\alpha \in S, m_{0} \in M_{0}, \alpha \varepsilon^{\prime} \delta m_{0}=\delta \alpha \varepsilon m_{0}=\delta \alpha m_{0}$. Hence

$$
v \delta \alpha m_{0}=v \alpha \varepsilon^{\prime} \delta m_{0}=v \alpha \delta m_{0}=v \delta m_{0} .
$$

Suppose that $n \geqq 3$, and let $k, l$ be distinct integers such that $1 \leqq k, l \leqq n-1$. Applying our formula with $\alpha=\tau_{l}, m_{0}=i_{k}-i_{n}$, we get

$$
v \delta\left(i_{k}-i_{n}\right)=v \delta\left(i_{k}-i_{l}\right)=v \delta\left(i_{k}-i_{n}\right)-v \delta\left(i_{l}-i_{n}\right) .
$$

Consequently $v \delta\left(i_{l}-i_{n}\right)=0$, i.e. $\delta\left(i_{l}-i_{n}\right) \in M_{0}$. But $M_{0}=\sum_{l=1}^{n-1} \Phi\left(i_{l}-i_{n}\right)$. Hence $\delta M_{0} \subset M_{0}$. 
Suppose finally that $n=2$ and that $\phi \xi_{1}^{\prime}=0, \phi \in \Phi_{0}$ imply that $\phi=0$. We have, writing $\delta\left(i_{1}-i_{2}\right)=\lambda_{1} i_{1}+\lambda_{2} i_{2}$,

$$
\begin{aligned}
\lambda_{1} i_{1}+\lambda_{2} i_{2} & =\delta\left(i_{1}-i_{2}\right)=\tau_{1} \varepsilon^{\prime} \delta \tau_{1} \varepsilon\left(i_{1}-i_{2}\right)=\tau_{1} \varepsilon^{\prime} \delta \tau_{1}\left(i_{1}-i_{2}\right)=-\tau_{1} \varepsilon^{\prime} \delta\left(i_{1}-i_{2}\right) \\
& =-\tau_{1} \varepsilon^{\prime}\left(\lambda_{1} i_{1}+\lambda_{2} i_{2}\right)=-\left(\lambda_{1} i_{2}+\lambda_{2} i_{1}\right)-\left(\xi_{1}^{\prime}-1\right)\left(\lambda_{1}+\lambda_{2}\right)\left(i_{1}-i_{2}\right) .
\end{aligned}
$$

Hence

$$
\left(\lambda_{1}+\lambda_{2}\right)\left\{2 i_{2}+\xi_{1}^{\prime}\left(i_{1}-i_{2}\right)\right\}=0 .
$$

Comparing coefficients we have

$$
2\left(\lambda_{1}+\lambda_{2}\right)=0, \quad\left(\lambda_{1}+\lambda_{2}\right) \xi_{1}^{\prime}=0 .
$$

Our hypothesis now gives $\lambda_{1}+\lambda_{2}=0$, i.e. $\delta\left(i_{1}-i_{2}\right) \in M_{0}$. The proof is complete.

Remark. The condition required for the case $n=2$ cannot be dispensed with in general. Consider for example the mapping $\delta$ defined by

$$
\delta\left(i_{1}-i_{2}\right)=2 i_{1}+2 i_{2}, \quad \delta i_{2}=0,
$$

taking for $\Phi$ the ring of the residue classes of the integers modulo 8. Clearly $\delta$ does not map $M_{0}$ into itself, and a straightforward calculation will show that $\delta \in \operatorname{End}_{\Gamma} \varepsilon M$ if $\varepsilon\left(\tau_{1}\right)=0$.

The next object is to determine the endomorphisms of $M_{0}$.

(4.7) LemMa. Every endomorphism of $M_{0}$ is a multiplication by a scalar.

Proof. Let $\delta \in \operatorname{End}_{\Gamma} M_{0}$ and put for $1 \leqq k \leqq n-1$,

$$
\delta\left(i_{k}-i_{n}\right)=\sum_{l=1}^{n-1} \phi\left(i_{k}, i_{l}\right)\left(i_{l}-i_{n}\right) \text {. }
$$

For any $\alpha \in S$ we have $\delta=\alpha^{-1} \delta \alpha$. Apply this, choosing for $\alpha$ any permutation leaving $i_{n}$ fixed. Then

$$
\begin{aligned}
\sum_{i=1}^{n-1} \phi\left(i_{k}, i_{l}\right)\left(i_{l}-i_{n}\right) & =\delta\left(i_{k}-i_{n}\right)=\alpha^{-1} \delta \alpha\left(i_{k}-i_{n}\right)=\alpha^{-1} \delta\left(\alpha i_{k}-i_{n}\right) \\
& =\alpha^{-1} \sum_{p=1}^{n-1} \phi\left(\alpha i_{k}, i_{p}\right)\left(i_{p}-i_{n}\right)=\sum_{l=1}^{n-1} \phi\left(\alpha i_{k}, \alpha i_{l}\right)\left(i_{l}-i_{n}\right) \quad\left(i_{p}=\alpha i_{l}\right) .
\end{aligned}
$$

Hence $\phi\left(i_{k}, i_{l}\right)=\phi\left(\alpha i_{k}, \alpha i_{l}\right)$ whenever $\alpha i_{n}=i_{n}$. This implies that, if $k \neq l ; k, l \leqq n-1$,

$$
\phi\left(i_{k}, i_{k}\right)=\phi\left(i_{1}, i_{1}\right)=\phi, \quad \phi\left(i_{k}, i_{1}\right)=\phi\left(i_{1}, i_{2}\right)=\chi,
$$

say. Consequently, writing $\phi-\chi=\zeta$,

$$
\delta\left(i_{k}-i_{n}\right)=\zeta\left(i_{k}-i_{n}\right)+\chi\left(e-n i_{n}\right) \quad(1 \leqq k \leqq n-1) .
$$

In the case $n=2$ this gives

$$
\delta\left(i_{1}-i_{2}\right)=\zeta\left(i_{1}-i_{2}\right)+\chi\left(i_{1}-i_{2}\right)=(\zeta+\chi)\left(i_{1}-i_{2}\right),
$$

so that the assertion is proved in this case. 
If $n>2$, let $p \leqq n-1$ and $p \neq k$. Since $\delta-\zeta$ commutes with $\tau_{p}$, we have

But

$$
(\delta-\zeta)\left(i_{k}-i_{p}\right)=(\delta-\zeta) \tau_{p}\left(i_{k}-i_{n}\right)=\tau_{p}(\delta-\zeta)\left(i_{k}-i_{n}\right)=\tau_{p} \chi\left(e-n i_{n}\right)=\chi\left(e-n i_{p}\right)
$$

$$
(\delta-\zeta)\left(i_{k}-i_{p}\right)=(\delta-\zeta)\left\{\left(i_{k}-i_{n}\right)-\left(i_{p}-i_{n}\right)\right\}=(\delta-\zeta)\left(i_{k}-i_{n}\right)-(\delta-\zeta)\left(i_{p}-i_{n}\right)=0 .
$$

Hence $\chi\left(e-n i_{p}\right)=0, \chi=0$, and $\delta\left(i_{k}-i_{n}\right)=\zeta\left(i_{k}-i_{n}\right)$. The result follows.

(4.8) COROLlaRY. The representation module $M_{0}$ is indecomposable.

This is an immediate consequence of (4.7) and (4.5).

(4.9) Lemma. Let $n>2$ and suppose that $\delta$ is a $\Gamma$-homomorphism of the representation module $\varepsilon M$ into the representation module $\varepsilon^{\prime} M$, where $\varepsilon$ is given by (3.3) and $\varepsilon^{\prime}$ by a similar formula having $\psi^{\prime}, \xi_{k}^{\prime}$ in place of $\psi, \xi_{k}$. Then we can find elements $\zeta, \theta, \theta_{0}$ of $\Phi$ such that for $m \in M$,

$$
\begin{gathered}
\delta m=\zeta m+v(m)\left\{\theta \sum_{1}^{n-1}\left(\xi_{k}^{\prime}-1\right) i_{k}+\theta_{0} e\right\}, \\
\theta \sum_{1}^{n-1} \xi_{k}^{\prime}=n\left(\theta-\theta_{0}\right), \quad \theta \psi^{\prime}=0 .
\end{gathered}
$$

Conversely, (4.10) defines a homomorphism $\delta$ provided that (4.11) are satisfied. These assertions are valid also in the case $n=2$ if no non-zero element of $\Phi_{0}$ annihilates $\xi_{1}^{\prime}$.

Proof. Let $n>2$. By (4.6), we have $\delta M_{0} \subset M_{0}$; i.e. $\delta$ induces an endomorphism of $M_{0}$. By (4.7), there exists $\zeta$ in $\Phi$ such that $\delta m=\zeta m$ whenever $m \in M_{0}$. Put $\Delta=\delta-\zeta$, so that $\Delta m=0$ whenever $m \in M_{0}$, and let $\Delta i_{n}=\sum_{1}^{n} \theta_{k} i_{k}$. The relation $\Delta=\tau_{k} \varepsilon^{\prime} \Delta \tau_{k} \varepsilon$ now gives

$$
\begin{aligned}
\sum_{1}^{n} \theta_{k} i_{k}=\tau_{k} \varepsilon^{\prime} \Delta \tau_{k} \varepsilon i_{n}=\tau_{k} \varepsilon^{\prime} \Delta\left\{i_{k}+\psi e+\left(\xi_{k}-1\right)\left(i_{k}-i_{n}\right)\right\} & =\tau_{k} \varepsilon^{\prime} \Delta\left\{i_{n}+\psi e+\xi_{k}\left(i_{k}-i_{n}\right)\right\}=\tau_{k} \varepsilon^{\prime} \Delta i_{n} \\
=\tau_{k} \varepsilon^{\prime} \sum_{1}^{n} \theta_{k} i_{k} & =\tau_{k} \sum_{1}^{n} \theta_{k} i_{k}+\left(\sum_{1}^{n} \theta_{k}\right)\left\{\psi^{\prime} e+\left(\xi_{k}^{\prime}-1\right)\left(i_{k}-i_{n}\right)\right\} .
\end{aligned}
$$

Let $l, k, n$ be different. Then comparing coefficients of $i_{l}, i_{k}$ we have, writing $\sum_{i}^{n} \theta_{k}=\theta$,

$$
\begin{gathered}
\dot{\psi}^{\prime} \theta=0 \\
\theta_{k}=\theta_{n}+\theta\left(\xi_{k}^{\prime}-1\right) \quad(1 \leqq k \leqq n-1) .
\end{gathered}
$$

Adding this over $k=1,2, \ldots, n-1$, we get

$$
\theta-\theta_{n}=(n-1) \theta_{n}+\theta \sum_{1}^{n-1} \xi_{k}^{\prime}-(n-1) \theta
$$

i.e. $\theta \sum_{1}^{n-1} \xi_{k}^{\prime}=n\left(\theta-\theta_{n}\right)$. Hence (4.11) is satisfied with $\theta_{0}=\theta_{n}$. Furthermore we have

$$
\Delta i_{n}=\sum_{1}^{n} \theta_{k} i_{k}=\sum_{1}^{n-1}\left[\theta_{0}+\theta\left(\xi_{k}^{\prime}-1\right)\right] i_{k}+\theta_{0} i_{n}=\theta_{0} e+\theta \sum_{1}^{n-1}\left(\xi_{k}^{\prime}-1\right) i_{k} .
$$


If now $m \in M$, then $m-v(m) i_{n} \in M_{0}$ and so

$$
\Delta m=v(m) \Delta i_{n}=v(m)\left\{\theta_{0} e+\theta \sum_{1}^{n-1}\left(\xi_{k}^{\prime}-1\right) i_{k}\right\} .
$$

This proves (4.10) and completes the proof of the direct part of the lemma in the case $n>2$. In view of the agreement that $\psi^{\prime}=0$ when $n=2$, we have also proved our assertion in this case.

Now we prove the converse assertion. We have to show that, if $\theta, \theta_{0}$ satisfy (4.11), then

$$
\Delta m=v(m)\left\{\theta_{0} e+\theta \sum_{i}^{n-1}\left(\xi_{k}^{\prime}-1\right) i_{k}\right\}=v(m) c,
$$

say, defines a homomorphism of $\varepsilon M$ into $\varepsilon^{\prime} M$. Let $\alpha$ be any permutation from $S$. Then, since $\alpha \varepsilon m \equiv \alpha m\left(\bmod M_{0}\right)$, we have $v(\alpha \varepsilon m)=v(\alpha m)=v(m)$. Hence

$$
\Delta \alpha \varepsilon m=\Delta m \text {. }
$$

On the other hand, for $1 \leqq k \leqq n-1$ we have

$$
\begin{aligned}
\tau_{k} \varepsilon^{\prime} \Delta m & =v(m) . \tau_{k} \varepsilon^{\prime} c=v(m)\left\{\tau_{k} c+v(c)\left[\psi^{\prime} e+\left(\xi_{k}^{\prime}-1\right)\left(i_{k}-i_{n}\right)\right]\right\} \\
& =v(m)\left\{\theta_{0} e+\theta \sum_{1}^{n-1}\left(\xi_{k}^{\prime}-1\right) i_{k}-\theta\left(\xi_{k}^{\prime}-1\right)\left(i_{k}-i_{n}\right)+v(c)\left[\psi^{\prime} e+\left(\xi_{k}^{\prime}-1\right)\left(i_{k}-i_{n}\right)\right]\right\} .
\end{aligned}
$$

But $v(c)=n \theta_{0}+\theta \sum_{1}^{n-1} \xi_{k}^{\prime}-\theta(n-1)=\theta$, by 4.11. Hence, as $\theta \psi^{\prime}=0$,

$$
\tau_{k} \varepsilon^{\prime} \Delta m=v(m) c=\Delta m .
$$

It follows that $\alpha \varepsilon^{\prime} \Delta m=\Delta m$ for all $\alpha \in S$, and the proof is complete.

(4.12) THEOREM. Every extension $\varepsilon M$ of $M_{0}$ by $\Phi$ is an indecomposable representation module with the exception of the split extension.

Proof. We have to treat the case $n=2$ separately. To begin with, assume that $n>2$.

Suppose that $\varepsilon M$ is a decomposable representation module. By (4.5), we can find an idempotent endomorphism $\delta$ of $\varepsilon M$ into itself such that $1, \delta$ are linearly independent. By (4.9) (with $\varepsilon=\varepsilon^{\prime}$ ), there exist elements $\zeta, \theta, \theta_{0}$ of $\Phi$ satisfying

$$
\theta \sum_{1}^{n-1} \xi_{k}=n\left(\theta-\theta_{0}\right), \quad \theta \psi=0
$$

such that

$$
\delta m=\zeta m+v(m)\left\{\theta \sum_{1}^{n-1}\left(\xi_{k}-1\right) i_{k}+\theta_{0} e\right\}=\zeta m+v(m) c
$$

say. Then $\zeta\left(i_{k}-i_{n}\right)=\delta\left(i_{k}-i_{n}\right)=\delta^{2}\left(i_{k}-i_{n}\right)=\zeta^{2}\left(i_{k}-i_{n}\right)$, whence $\zeta$ is an idempotent element of $\Phi$. Next, writing $\Delta=\delta-\zeta$, we have $\Delta m=v(m) c$,

$$
\Delta^{2} m=v(m) \Delta c=v(m) v(c) c=\theta v(m) c .
$$


Hence $\Delta^{2}=\theta \Delta$; i.e. $(\delta-\zeta)^{2}=\theta(\delta-\zeta)$. But $\delta^{2}=\delta, \zeta^{2}=\zeta$. Consequently

$$
(1-2 \zeta-\theta) \delta+\zeta(1+\theta)=0 \text {. }
$$

However $\delta, 1$ are linearly independent over $\Phi$. Hence

$$
\theta=1-2 \zeta, \quad \theta^{2}=(1-2 \zeta)^{2}=1-4 \zeta+4 \zeta^{2}=1 .
$$

By (4.13), we have

$$
\sum_{1}^{n-1} \xi_{k}=\theta^{2} \sum_{1}^{n-1} \xi_{k}=n \theta\left(\theta-\theta_{0}\right) \in n \Phi
$$

and $\psi=\theta^{2} \psi=\theta \cdot \theta \psi=0$. Accordingly $\varepsilon$ is a coboundary and the extension $\varepsilon M$ is a split extension. This completes the proof in the case $n>2$.

It remains to prove the theorem for $n=2$. Suppose that the representation module $\varepsilon M$ is decomposable, and let $M=X+X^{\prime}$ be a decomposition of $M$ into a direct sum of proper representation submodules. Since any $\Phi$-base of $M$ has exactly two elements, the submodules $X, X^{\prime}$ have bases of a single element each. Let $x, x^{\prime}$ be such elements. Then $x, x^{\prime}$ form a $\Phi$-base for $M$. Put

$$
\left[\begin{array}{c}
i_{1}-i_{2} \\
i_{2}
\end{array}\right]=\left[\begin{array}{cc}
\alpha & \alpha^{\prime} \\
\beta & \beta^{\prime}
\end{array}\right]\left[\begin{array}{l}
x \\
x^{\prime}
\end{array}\right]
$$

The 2 by 2 matrix in this equation is invertible, and the same must also be true of its determinant $\alpha \beta^{\prime}-\alpha^{\prime} \beta=\mu$, say. Applying $\tau_{1} \varepsilon$ to both sides of the above equation, we have

$$
\begin{aligned}
{\left[\begin{array}{ll}
\alpha & \alpha^{\prime} \\
\beta & \beta^{\prime}
\end{array}\right]\left[\begin{array}{c}
\tau_{1} \varepsilon x \\
\tau_{1} \varepsilon x^{\prime}
\end{array}\right] } & =\left[\begin{array}{c}
-\left(i_{1}-i_{2}\right) \\
\xi_{1}\left(i_{1}-i_{2}\right)+i_{2}
\end{array}\right]=\left[\begin{array}{cc}
-1 & 0 \\
\xi_{1} & 1
\end{array}\right]\left[\begin{array}{c}
i_{1}-i_{2} \\
i_{2}
\end{array}\right] \\
& =\left[\begin{array}{cc}
-1 & 0 \\
\xi_{1} & 1
\end{array}\right]\left[\begin{array}{ll}
\alpha & \alpha^{\prime} \\
\beta & \beta^{\prime}
\end{array}\right]\left[\begin{array}{l}
x \\
x^{\prime}
\end{array}\right] .
\end{aligned}
$$

Premultiplying by

$$
\left[\begin{array}{rr}
\beta^{\prime} & -\alpha^{\prime} \\
-\beta & \alpha
\end{array}\right]
$$

we find that

$$
\mu\left[\begin{array}{c}
\tau_{1} \varepsilon x \\
\tau_{1} \varepsilon x^{\prime}
\end{array}\right]=\left[\begin{array}{cc}
-\alpha \alpha^{\prime} \xi_{1}-\alpha \beta^{\prime}-\alpha^{\prime} \beta & -\alpha^{\prime^{2}} \xi_{1}-2 \alpha^{\prime} \beta^{\prime} \\
\alpha^{2} \xi_{1}+2 \alpha \beta & \alpha \alpha^{\prime} \xi_{1}+\alpha \beta^{\prime}+\alpha^{\prime} \beta
\end{array}\right]\left[\begin{array}{c}
x \\
x^{\prime}
\end{array}\right] .
$$

Since $X, X^{\prime}$ are $\Gamma$-submodules and their sum is direct, the 2 by 2 matrix in this relation must be diagonal. Hence

$$
\alpha^{2} \xi_{1}=-2 \alpha \beta, \quad \alpha^{\prime 2} \xi_{1}=-2 \alpha^{\prime} \beta^{\prime}
$$

and so

$$
\mu^{2} \xi_{1}=\left(\alpha \beta^{\prime}-\alpha^{\prime} \beta\right)^{2} \xi_{1} \equiv{\beta^{\prime}}^{2} \alpha^{2} \xi_{1}+\beta^{2} . \alpha^{\prime 2} \xi_{1} \equiv 0 \quad(\bmod 2 \Phi) .
$$

However $\mu^{2}$ is invertible. Hence $\xi_{1} \in 2 \Phi$ and the extension $\varepsilon M$ splits. The proof is now complete. 
5. An irreducible modular representation. In this section $\Phi$ will denote a field of characteristic $p$ (possibly zero). Our object is to obtain the irreducible constituents of the matrix representation of $S$ obtained from the representation module $M_{0}$. We begin with a preliminary result. The coefficient of $i$ in $x$ will be denoted by $(x)_{i}$.

(5.1) Lemma. If $x$ is any element of $M_{0}$ and $i$ any element of $E$, then

$$
(x)_{i}(n i-e) \in \Gamma x \text {. }
$$

Here $e$ denotes the sum of the elements of $E$.

Proof. Let $E^{\prime}$ denote the set of all elements of $E$ other than $i$, and let $\sigma$ be any permutation of $E$ leaving $i$ fixed and permuting the elements of $E^{\prime}$ cyclically. Let

$$
x^{\prime}=\sum_{u \in E^{\prime}}(x)_{u} u,
$$

so that $x=x^{\prime}+(x)_{i} i, v(x)=v\left(x^{\prime}\right)+(x)_{i}$. Then clearly

$$
\left(1+\sigma+\sigma^{2}+\ldots+\sigma^{n-2}\right) x=v\left(x^{\prime}\right)(e-i)+(n-1)(x)_{i} i=(x)_{i}(n i-e),
$$

since $v(x)=0$. The lemma follows.

(5.2) THEOREM. If $\Phi$ is a field of characteristic $p$ not dividing $n$, the matrix representation (of degree $n-1$ ) of $S$ obtained from the representation module $M_{0}$ is irreducible. If on the other hand $p$ divides $n$, this representation contains an irreducible constituent of degree $n-2$, the remaining constituent being the unit representation.

Proof. (i) Suppose firstly that $p$ does not divide $n$, and let $x$ be a non-zero element of $M_{0}$. Then, by (5.1), we have

$$
(x)_{i}(n i-e) \in \Gamma x \quad(\text { for all } i \in E) .
$$

But $(x)_{i} \neq 0$ for some $i \in E$ and $\Phi$ is a field. Hence $n i-e \in \Gamma x$ for some $i \in E$. If now $j$ is any element of $E$, and $\tau$ is the 2-cycle $(i j)$ then

$$
n j-e=\tau(n i-e) \in \Gamma x .
$$

Since in this case $n \Phi=\Phi$, we get for $j, k \in E$,

$$
j-k=n^{\prime}[(n j-e)-(n k-e)] \in \Gamma x,
$$

where $n n^{\prime}=1$. But the elements $j-k$ generate the $\Phi$-module $M_{0}$. Hence $M_{0} \subset \Gamma x$; and so $\Gamma x=M_{0}$ for every non-zero element $x$ of $M_{0}$. The representation module $M_{0}$ is therefore simple, and the corresponding matrix representation is irreducible.

(ii) Assume now that $p$ divides $n$. Then $v(e)=n .1=0$; that is $e \in M_{0}$, and obviously $\Phi e$ is a representation submodule of $M_{0}$. As such $\Phi e$ is isomorphic with $\Phi$ and so gives rise to the unit representation. We shall show that the factor module $M_{0} / \Phi e$ is a simple representation module.

Let $i$ be a fixed element of $E$, let $E^{\prime}$ be the set of all elements of $E$ other than $i$, and denote by $S^{\prime}$ the subgroup of $S$ consisting of those permutations of $S$ leaving $i$ fixed. We identify 
$S^{\prime}$ with the symmetric group on $E^{\prime}$. Since the elements $\left\{k-i ; k \in E^{\prime}\right\}$ form a $\Phi$-base for $M_{0}$, there is a unique $\Phi$-isomorphism $\theta$ of $M_{0}$ onto $M^{\prime}=\sum_{k \in E^{\prime}} \Phi k$, for which $\theta(k-i)=k$ for all $k$ in $E^{\prime}$. We observe however that $\theta$ is in fact an $S^{\prime}$-homomorphism. Furthermore we have, since $n .1=0$,

$$
\theta(e)=\theta \sum_{k \in E^{\prime}}(k-i)=\sum_{k \in E^{\prime}} k=e^{\prime},
$$

say. Hence the $\Gamma^{\prime}$-isomorphism $\theta$ carries $\Phi e$ onto $\Phi e^{\prime}$ (here $\Gamma^{\prime}=\Phi\left(S^{\prime}\right)$ is the group algebra over $\Phi$ of $S^{\prime}$ and $\Gamma^{\prime} \subset \Gamma$ ). It follows that $\theta$ induces a $\Gamma^{\prime}$-isomorphism of the factor module $M_{0} / \Phi e$ onto the factor module $M^{\prime} / \Phi e^{\prime}$. However the number of elements of $E^{\prime}$, namely $n-1$, is not divisible by $p$. The $\Gamma^{\prime}$-module $M^{\prime}$ therefore splits into the direct sum of $\Phi e^{\prime}$ and $M_{0}^{\prime}=M^{\prime} \cap M_{0}^{\prime}$ (cf. (2.1)), and, by the first part of this proof, $M_{0}^{\prime}$ is a simple $\Gamma^{\prime}$-module. As $M^{\prime} / \Phi e^{\prime}$ is then isomorphic with $M_{0}^{\prime}$, it follows that $M_{0} / \Phi e$ is a simple $\Gamma^{\prime}$-module. Hence, for any non-zero element $x$ of $M_{0} / \Phi e$ we have

$$
M_{0} / \Phi e \subset \Gamma^{\prime} x \subset \Gamma x,
$$

and so $M_{0} / \Phi e=\Gamma x$; that is $M_{0} / \Phi e$ is a simple $\Gamma$-module. The proof of the theorem is complete.

We end this section by summarising our results in the case of a field $\Phi$ of characteristic $p$.

(i) If $p$ is not a factor of $n$ we have $n \Phi=\Phi$ and so (by (3.5)) all the extensions of $M_{0}$ by $\Phi$ are equivalent to the extension $M$. Furthermore $M_{0}$ itself is a simple representation module and so $M \supset M_{0} \supset 0$ is a composition series for $M$.

(ii) If $p$ is a factor of $n$ we have $n \Phi=0$, and there are " plenty " of extensions of $M_{0}$ by $\Phi$, all of which (with the exception of the split extension) are indecomposable representation modules. The module $M_{0}$ is indecomposable but not simple. In fact we have a composition series

$$
\varepsilon M \supset M_{0} \supset \Phi e \supset 0
$$

for each of the modules $\varepsilon M$.

Note that our analysis has yielded an irreducible modular representation for each symmetric group, of degree $n-1$ or $n-2$. In point of fact we get another irreducible representation (except when $p=2$ ) by constructing the "associated" representation, i.e. by changing the sign of every matrix representing an odd permutation.

6. In this final section we investigate the symmetric group $S$ on an infinite set $E$. The results will be simpler than in the finite case.

(6.1) Lemma. Let $\Phi$ be any commutative ring and $M$ a free $\Phi$-module with base an infinite set $E$. Then the only endomorphisms of the representation module $M$ of the restricted symmetric group $S$ on $E$ are multiplications by elements of $\Phi$.

Proof. Let $\delta$ be any endomorphism of $M$, and for each $i \in E$ write

$$
\delta i=\sum_{i \in E} \delta(i, j) j,
$$


where the coefficients $\delta(i, j)$ are almost all zero. If $\alpha \in S$ then $\alpha \delta=\delta \alpha$; i.e. $\alpha^{-1} \delta \alpha=\delta$. Hence for each $i \in E$ we have

$$
\sum_{j \in E} \delta(i, j) j=\delta i=\alpha^{-1} \delta \alpha i=\alpha^{-1} \sum_{k \in E} \delta(\alpha i, k) k=\sum_{k \in E} \delta(\alpha i, k) \cdot \alpha^{-1} k .
$$

Comparing coefficients of $j$, we get

$$
\delta(i, j)=\delta(\alpha i, \alpha j)
$$

for all $i, j \in E$ and all $\alpha \in S$. Since $\delta(i, j)=0$ for almost all $j$ and $E$ is infinite, we can find $j_{i}$, different from $i$, such that $\delta\left(i, j_{i}\right)=0$. If now $i \neq j$, there exists a permutation $\alpha$ in $S$ such that

$$
\alpha(i)=i, \quad \alpha(j)=j_{i} .
$$

Using such a permutation in (6.2), we get

$$
\delta(i, j)=0 \text { whenever } i \neq j .
$$

Once more, if $i, i^{\prime}$ are any elements of $E$ and $\alpha$ any permutation carrying $i$ to $i^{\prime}$, equation (6.2) gives

$$
\delta(i, i)=\delta\left(i^{\prime}, i^{\prime}\right)=\phi,
$$

say. We have thus proved that $\delta i=\phi i$ for all $i \in E$, and so $\delta m=\phi m$ for all $m \in M$. This completes the proof.

(6.2) THEOREM. The natural representation module $M$ of an infinite symmetric group is indecomposable over any commutative ring.

This follows at once from (6.1) and (4.5).

Again let $v: M \rightarrow \Phi$ be defined by

$$
v\left(\sum_{i \in E} \phi_{i} i\right)=\sum_{i \in E} \phi_{i}
$$

and let $M_{0}$ be the kernel of $v$. As before $M_{0}$ is a representation submodule of $M$. We prove

(6.3) THEOREM. If $\Phi$ is any field then the representation module $M_{0}$ of the infinite symmetric group $S$ is simple.

Proof. Let $\Gamma=\Phi(S)$ be the group algebra of $S$ over $\Phi$. We must show that $M_{0} \subset \Gamma x$ for every non-zero element $x$ of $M_{0}$. The coefficient of $i$ in $x$ will be denoted by $(x)_{i}$. Let $D$ denote the (finite) non-empty subset of $E$ consisting of all $i$ in $E$ such that $(x)_{i} \neq 0$, and let $d$ be the number of elements of $D$. Choose a fixed element, say $u$, of $D$. Note that necessarily $d \geqq 2$ because $D$ is non-empty and $\sum_{i \in D}(x)_{i}=0$. If $D^{\prime}$ is the set of elements of $D$ other than $u$, and $\sigma$ is a cycle on $D^{\prime}$ leaving all other elements fixed, then easily

$$
\left(1+\sigma+\sigma^{2}+\ldots+\sigma^{d-2}\right) x=\left\{\sum_{i \in D^{\prime}}(x)_{i}\right\}\left\{\sum_{i \in D^{\prime}} i\right\}+(d-1)(x)_{u} u=(x)_{\mu}\left(d u-\sum_{i \in D} i\right) .
$$

As $(x)_{u} \neq 0$ and $\Phi$ is a field, we conclude that $d u-\sum_{i \in D} i$ belongs to $\Gamma x$. We have thus proved: 
(6.4) If $x=\sum_{i \in D}(x)_{i} i \in M_{0}$ and $(x)_{i} \neq 0$ (all $i \in D$ ), then, for any $u \in D$, we have

$$
d u-\sum_{i \in D} i \in \Gamma x
$$

where $d=|D|$.

Now to complete the proof, let $x \neq 0$ be as above, and let $a, b$ be any two distinct elements of $E$. The proof will be complete if we show that $b-a \in \Gamma x$, because the elements $b-a$ generate $M_{0}$ as $\Phi$-module. Let $K$ be any subset of $E$ having exactly $d-1$ elements and disjoint from $\{a, b\}$ Since $d \geqq 2, K$ is non-empty. Choose an element $k$ of $K$. Put

$$
A=K \cup\{a\}, \quad B=K \cup\{b\} ;
$$

thus $|A|=|B|=d$ and there exist permutations $\alpha, \beta$ in $S$ such that

$$
\alpha u=k, \quad \beta u=k, \alpha D=A, \quad \beta D=B .
$$

Applying (6.4) to $\alpha x$ in place of $x$, we conclude that $d k-\sum_{i \in A} i \in \Gamma \alpha x=\Gamma x$. In the same way we have $d k-\sum_{i \in B} i \in \Gamma x$, and therefore their difference $\sum_{i \in B} i-\sum_{i \in A} i=b-a$ also belongs to $\Gamma x$. This completes the proof.

The UNIVERSITY

SHEFFIELD 